\title{
ASSOCIAÇÃO ENTRE A CINETOSE E ASPECTOS DO DESENVOLVIMENTO MOTOR EM CRIANÇAS DE UMA ESCOLA DO EXTREMO SUL CATARINENSE
}

The association between motion sickness and aspects of motor development in children of a school in the South of Santa Catarina

Associação entre cinetose e desenvolvimento motor Association between motion sickness and motor development

Évelin Vicente, Morgana Teixeira Magnus, Pâmela Selau Bittencourt

Autor correspondente: Évelin Vicente

Endereço profissional:

Curso de Fisioterapia - Universidade do Extremo Sul Catarinense.

Av. Universitária, 1105 Bairro: Pinheirinho, Criciúma - SC

CEP: 88806-000

Email: eve@unesc.net

\section{RESUMO}

O objetivo do estudo foi avaliar a associação entre a cinetose e aspectos do desenvolvimento motor em crianças de uma escola do Extremo Sul Catarinense. Estudo de abordagem quantitativa e corte transversal com escolares da rede fundamental de ensino com idade entre 8 e 11 anos. Para o levantamento de cinetose foi utilizado o questionário Motion Sickness Susceptibility Questionnaire Short-form (MSSQ-short) e para avaliar o desenvolvimento motor dos escolares foi utilizado o Protocolo de testes da Escala de Desenvolvimento Motor - EDM. Das 21 crianças avaliadas, 15 demonstraram presença de cinetose em pelo menos um meio de transporte ou entretenimento, sendo $100 \%$ na idade de 8 anos e $90 \%$ no sexo feminino. Os meios de transportes com maiores índices de cinetose foram ônibus ou vans (9 crianças) e carros (6 crianças). Não houve associação estatisticamente significativa entre a presença de cinetose e aspectos do desenvolvimento motor. Principais alterações ocorreram no âmbito de equilíbrio (46,7\%) e motricidade global (40\%). Foi possível verificar que a maioria das crianças do estudo apresentaram cinetose. Não foi possível estabelecer uma associação entre a presença de cinetose e alterações do desenvolvimento motor.

Palavras-chaves: Cinetose; Desenvolvimento infantil; Crianças. 


\section{ABSTRACT}

The objective of this study was to evaluate the association between motion sickness and aspects of motor development in children of a school in the South of Santa Catarina. A quantitative and cross-sectional study with schoolchildren aged 8 to 11 years. The Motion Sickness Susceptibility Questionnaire Short-form (MSSQ-short) questionnaire was used to assess the motor development of schoolchildren using the Motor Development Scale (EDM) Test Protocol. Of the 21 children evaluated, 15 demonstrated the presence of motion sickness in at least one means of transport or entertainment, being $100 \%$ at the age of 8 years and $90 \%$ in the female sex. The means of transport with higher rates of kinesis were buses or vans ( 9 children) and cars (6 children). There was no statistically significant association between the presence of motion sickness and aspects of motor development. Main changes occurred in the balance $(46.7 \%)$ and global motor $(40 \%)$. It was possible to verify that the majority of the children of the study presented motion sickness. It was not possible to establish an association between the presence of motion sickness and changes in motor development.

Keywords: Motion sickness; Child development; Child.

\section{Introdução}

A cinetose é considerada uma vertigem fisiológica e, desta forma, não é considerada uma doença, mas sim uma resposta comum a uma situação anormal. ${ }^{1}$ É causada por certos tipos de movimento e é induzida durante a locomoção passiva em veículos, gerada por acelerações desconhecidas do corpo, às quais a pessoa não se adaptou, ou por um conflito intersensorial entre estímulos vestibulares e visuais. ${ }^{2,3}$ É identificada por meio do questionário Motion Sickness Susceptibility Questionnaire Short-form (MSSQ-short), um método padrão não-invasivo para medir a susceptibilidade da cinetose. ${ }^{1}$

Seus sintomas incluem náuseas, vômito, palidez, suor frio, hipersalivação, hiperventilação e cefaleia, ocorrendo frequentemente durante viagens de automóveis, trens, aviões ou navios ou sempre que o indivíduo que sofre desta situação está em movimento, como em um carrossel ou balanço do parque. ${ }^{4}$

Embora várias hipóteses tenham sido propostas para explicar o conjunto de sintomas da cinetose, o mecanismo biológico preciso da diferença individual na 
susceptibilidade à mesma ainda não foi esclarecido. ${ }^{5}$ Tem sido demonstrado que quase todos os indivíduos saudáveis podem desenvolver a cinetose, quando expostos ao movimento provocativo apropriado. A prevalência depende da estimulação e do limiar individual para o movimento e varia em diferentes situações, o que torna difícil prever sua ocorrência. ${ }^{5,6}$

O acúmulo de evidências indica que sexo e idade são dois preditores principais da susceptibilidade à cinetose na população em geral. ${ }^{7} \mathrm{Um}$ dos fenômenos mais comumente observados é que as mulheres são mais suscetíveis do que os homens. ${ }^{8}$ Um estudo prévio com 831 crianças entre 7 e 12 anos demonstrou escores maiores de MSSQ-short em meninas. ${ }^{9}$ Os picos de susceptibilidade ocorrem em torno de 9 a 10 anos e, posteriormente, diminuem a partir da adolescência para a idade adulta. ${ }^{9,10}$

Indivíduos que possuem suscetibilidade a cinetose apresentam uma redução nas funções cognitivas, como demonstrado pelo baixo desempenho em diferentes tarefas psicomotoras. ${ }^{11}$ Como em adultos, as repercussões da cinetose em crianças afetam as atividades da vida diária e podem levar à deficiência e isolamento social, que exercem um efeito negativo e impacto em diferentes aspectos do desenvolvimento. ${ }^{12,13}$

Inúmeros são os fatores que podem por em risco o desenvolvimento normal de uma criança, entre eles uma série de fatores biológicos ou ambientais aumentam as possibilidades de deficiências no desenvolvimento neuropsicomotor na infância. ${ }^{14}$ Os primeiros anos de escolaridade são um período crítico no desenvolvimento cognitivo e motor da criança, durante o qual são adquiridas competências que podem afetar um vasto leque de funções. ${ }^{15}$

Considerando os elevados picos de susceptibilidade de cinetose em crianças em idade escolar descritos e as possíveis relações dessa com o desenvolvimento motor, além da carência de estudos englobando esses aspectos, esse estudo objetivou levantar dados a respeito desse tema em escolares de uma escola do município de Criciúma (SC).

\section{Materiais e Métodos}

Este é um estudo de abordagem quantitativa e corte transversal. Para sua execução, foram seguidos todos os aspectos éticos preconizados pela Resolução 466/2012 do Conselho Nacional de Saúde. O estudo foi aprovado pelo Comitê de Ética em Pesquisa da Universidade do Extremo Sul Catarinense (UNESC) sob o parecer número 2.083.994, sendo os escolares incluídos somente após assinatura do Termo de Consentimento Livre e Esclarecido (TCLE) pelos pais ou responsáveis, que foram previamente esclarecidos quanto aos objetivos e procedimentos, com livre acesso aos questionários de avaliação propostos.

A coleta dos dados foi realizada no Colégio Unesc, localizado na cidade de Criciúma, Santa Catarina, no período de agosto a setembro de 2017, com uma amostra de escolares da rede fundamental de ensino com idade entre 8 e 11 anos. A 
escola apresenta cerca de 100 alunos dentro desta faixa etária, tendo todos os pais ou responsáveis sido convidados a autorizar a participação dos mesmos no estudo. Foram incluídos escolares dentro dessa faixa etária, devidamente matriculados na escola e que possuíam o consentimento dos pais e/ou responsáveis por meio da assinatura do TCLE e excluídos os que apresentavam algum diagnóstico que levasse a alteração motora e/ou intelectual.

As variáveis estudadas incluíram aspectos demográficos, susceptibilidade à cinetose e alterações do desenvolvimento motor.

\section{Susceptibilidade à cinetose}

Para o levantamento de cinetose foi utilizado o questionário MSSQ-short. Desenvolvido por Reason e Brand em 1975, ${ }^{16}$ o MSSQ-short é considerado um método padrão não-invasivo, quantificado, de fácil execução e validado para avaliar a susceptibilidade à cinetose. ${ }^{7}$ Nesse estudo foi utilizada a versão adaptada culturalmente e traduzida para o português do Brasil por França e Branco-Barreiro em $2013 .{ }^{17}$

O MSSQ-short investiga quais movimentos são responsáveis por iniciar os sintomas da cinetose, considerando nove tipos distintos de meios de locomoção e entretenimentos que podem gerar os sintomas. ${ }^{18}$ Possui dois escores, A e B. O escore $A$ avalia movimentos de susceptibilidade a doença antes da idade de 12 anos e o $\mathrm{B}$ corresponde à susceptibilidade a doença de movimento durante os 10 anos anteriores à consulta.19 Para o estudo foram utilizadas apenas as questões relacionadas à presença da cinetose na infância, o que corresponde somente ao escore A (antes dos 12 anos). Para cada meio de transporte ou entretenimento a criança respondeu, juntamente com auxílio dos pais ou responsáveis: "nunca utilizei", "nunca ficava enjoado", "raramente ficava enjoado", "às vezes ficava enjoado" ou "sempre ficava enjoado". Assim como no estudo de França et al. ${ }^{18}$, para a análise da susceptibilidade à cinetose a resposta "nunca ficava enjoado" foi considerada não ter cinetose e as respostas: "raramente ficava enjoado", "às vezes ficava enjoado" e, "sempre ficava enjoado" foram agrupadas como presença de cinetose.

\section{Avaliação do desenvolvimento motor}

Após o levantamento de dados sobre cinetose, foram aplicados testes de avaliação do desenvolvimento motor tanto nas crianças com queixas de cinetose quanto nas que não apresentavam. $O$ instrumento utilizado foi o protocolo de testes da Escala de Desenvolvimento Motor (EDM) desenvolvido por Neto. ${ }^{20} \mathrm{~A}$ EDM atinge um conjunto de evidências diversificadas e de dificuldades graduadas, sendo cada uma delas próprias a uma idade cronológica. Engloba avaliações das áreas do desenvolvimento compreendidas em motricidade fina, motricidade global, equilíbrio, esquema corporal, organização espacial e temporal e lateralidade. As avaliações são distribuídas entre 2 a 11 anos e consistem em 10 tarefas motoras cada, exceto nos 
testes de lateralidade onde as tarefas são iguais para todas as faixas etárias. ${ }^{21}$ Como no estudo, as crianças apresentam idades entre 8 e 11 anos, a avaliação foi realizada usando os testes equivalentes a idade em questão. Foram consideradas alterações nas funções motoras, a falha na execução dos testes das áreas do desenvolvimento de forma isolada.

\section{Análise dos dados}

Posteriormente, os dados foram tabulados e analisados no programa Statistical Package for the Social Sciences - SPSS, versão 22.0 e expressos em média e desvio padrão ou frequência e percentual. As associações entre as variáveis foram analisadas por meio do teste exato de Fisher, sendo o nível de significância considerado menor que $5 \%$.

\section{Resultados}

Do total de 100 crianças cujos pais ou responsáveis foram contatados, houve retorno de 25. Destes, 4 pais negaram a participação dos filhos no estudo, resultando em uma amostra de 21 crianças. A idade média das crianças foi de 9,86 \pm 1,06 anos, sendo $52,4 \%$ do sexo feminino. De todas as crianças entrevistadas, 15 possuíam presença de cinetose em pelo menos um meio de transporte ou entretenimento. Durante a avaliação do desenvolvimento motor as maiores alterações ocorreram nos aspectos de equilíbrio e motricidade global, respectivamente (Tabela 1). Não foram observadas alterações no aspecto lateralidade.

Tabela 1. Aspectos demográficos, alterações do desenvolvimento motor e frequência de cinetose em crianças de uma escola de ensino fundamental. Criciúma (SC), 2017.

\begin{tabular}{lc}
\hline Variáveis & $\begin{array}{c}\text { Média } \pm \text { Desvio padrão ou n (\%) } \\
\mathrm{n}=21\end{array}$ \\
\hline Idade (anos) & $9,86 \pm 1,06$ \\
Sexo & \\
Feminino & $11(52,4)$ \\
Masculino & $10(47,6)$ \\
Cinetose & \\
Sim & $15(71,4)$ \\
Não & $6(28,6)$ \\
Alterações* & \\
Motricidade fina & \\
Motricidade global & $4(19,7)$ \\
\end{tabular}


Equilíbrio

$9(42,9)$

Esquema corporal

$5(23,8)$

Organização temporal

$3(14,3)$

Organização espacial

${ }^{*}$ Crianças que não realizaram os testes adequadamente.

Os resultados obtidos, através da aplicação do questionário MSSQ-Short, demonstram que os meios de transportes, os quais apresentaram maiores índices de cinetose (levando em consideração as respostas "raramente ficava enjoado", "às vezes ficava enjoado" e "sempre ficava enjoado"), foram ônibus ou vans (9 crianças) e carros (6 crianças), respectivamente. No quesito entretenimento, o gira-gira de parquinhos (11 crianças) foi o mais frequente, seguido de brinquedos em parques de diversão (6 crianças) (Tabela 2$)$. 
Tabela 2. Frequência de susceptibilidade à cinetose em crianças de uma escola de ensino fundamental, segundo o meio de transporte ou de entretenimento. Criciúma (SC), 2017.

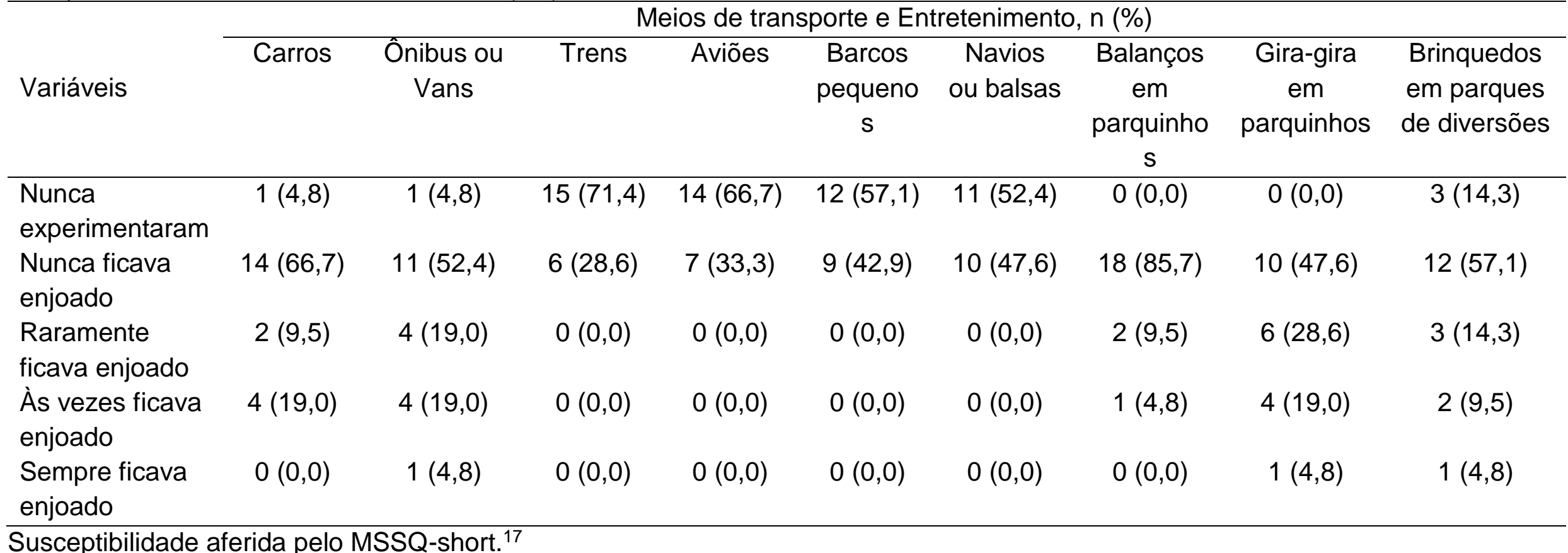


Foi possível identificar que a presença de cinetose foi mais frequente na idade de 8 anos (100\%) e no sexo feminino (90\%) (Tabela 3).

Tabela 3. Relação entre cinetose, idade e sexo em crianças de uma escola de ensino fundamental. Criciúma (SC), 2017.

\begin{tabular}{|c|c|c|}
\hline Variáveis & Cinetose & Valor-p \\
\hline \multicolumn{3}{|l|}{ Idade (anos) } \\
\hline \multicolumn{3}{|l|}{ Oito $(n=3)$} \\
\hline Sim & $3(100)$ & \\
\hline Não & $0(0)$ & \\
\hline Nove $(n=4)$ & & $0,442^{*}$ \\
\hline Sim & $3(80)$ & \\
\hline Não & $1(20)$ & \\
\hline \multicolumn{3}{|l|}{$\operatorname{Dez}(n=7)$} \\
\hline Sim & $4(57,14)$ & \\
\hline Não & $3(42,85)$ & \\
\hline \multicolumn{3}{|l|}{ Onze $(n=7)$} \\
\hline Sim & $5(71,42)$ & \\
\hline Não & $2(28,57)$ & \\
\hline \multicolumn{3}{|l|}{ Sexo } \\
\hline Feminino $(\mathrm{n}=11)$ & & $0,063^{* *}$ \\
\hline Sim & $10(90,9)$ & \\
\hline Não & $1(9,09)$ & \\
\hline \multicolumn{3}{|l|}{ Masculino $(n=10)$} \\
\hline Sim & $5(50,0)$ & \\
\hline Não & $5(50,0)$ & \\
\hline
\end{tabular}

${ }^{\star}$ Razão verossimilhança

${ }^{* *}$ Teste exato de Fisher

Apesar de não haver significância estatística entre a presença de cinetose e aspectos do desenvolvimento motor, pode-se observar que as maiores alterações ocorreram no âmbito de equilíbrio $(46,7 \%)$ e motricidade global $(40 \%)$, mas não representaram a maioria dos casos. As crianças sem queixas de cinetose, também apresentaram algumas alterações como demonstra a Tabela 4.

Tabela 4. Associação entre a presença de cinetose e aspectos do desenvolvimento motor em crianças de uma escola de ensino fundamental. Criciúma (SC), 2017.

\begin{tabular}{lccc}
\hline \multirow{2}{*}{ Variáveis } & \multicolumn{2}{c}{ Cinetose } & \multirow{2}{*}{ Valor-p } \\
\cline { 2 - 3 } & Sim, $\mathrm{n}=15$ & Não, $\mathrm{n}=6$ & \\
\cline { 2 - 3 } $\begin{array}{l}\text { Motricidade fina } \\
\text { Realizou }\end{array}$ & $12(80,0)$ & $5(83,3)$ & $0,999^{\star}$ \\
$\quad$ Não realizou & $3(20,0)$ & $1(16,7)$ & \\
$\begin{array}{l}\text { Motricidade global } \\
\text { Realizou }\end{array}$ & & & \\
\hline \hline
\end{tabular}

Revista Inova Saúde, Criciúma, vol. 9, n. 2, jul. 2019.

ISSN 2317-2460 
Artigo Original

Atenção à saúde

\begin{tabular}{|c|c|c|c|}
\hline Não realizou & $6(40,0)$ & $2(33,3)$ & \\
\hline \multicolumn{4}{|l|}{ Equilíbrio } \\
\hline Realizou & $8(53,3)$ & $4(66,7)$ & \multirow[t]{2}{*}{$0,659^{*}$} \\
\hline Não realizou & $7(46,7)$ & $2(33,3)$ & \\
\hline \multicolumn{4}{|c|}{ Esquema corporal } \\
\hline Realizou & $10(66,7)$ & $6(100,0)$ & \multirow[t]{2}{*}{$0,262^{*}$} \\
\hline Não realizou & $5(33,3)$ & $0(0,0)$ & \\
\hline \multicolumn{4}{|c|}{ Drganização temporal } \\
\hline Realizou & $12(80,0)$ & $6(100,0)$ & \multirow[t]{2}{*}{$0,526^{*}$} \\
\hline Não realizou & $3(20,0)$ & $0(0,0)$ & \\
\hline \multicolumn{4}{|l|}{ _ateralidade } \\
\hline Realizou & $15(100,0)$ & $6(100,0)$ & - \\
\hline \multicolumn{4}{|c|}{ Drganização espacial } \\
\hline Realizou & $13(86,7)$ & $3(50,0)$ & \multirow[t]{2}{*}{$0,115^{*}$} \\
\hline Não realizou & $2(13,3)$ & $3(50,0)$ & \\
\hline
\end{tabular}

*Teste exato de Fisher

\section{Discussão}

O estudo teve como intuito identificar crianças que apresentavam cinetose e associar com possíveis alterações nos aspectos do desenvolvimento motor.

No presente estudo, encontrou-se maior suscetibilidade à cinetose, nas idades de 8 anos (100\% das crianças nesta faixa etária) e 9 anos ( $80 \%$ das crianças nesta faixa etária) e no sexo feminino (90\%), o que corrobora com um estudo que avaliou 25 alunos do ensino fundamental $(12$ meninos e 13 meninas, faixa etária = 9,8-11,7 anos, idade média $=10,60$ ) sendo que destas, as meninas apresentaram uma incidência de $76,92 \%$ enquanto os meninos de $33,33 \% .{ }^{22}$ Outro estudo que avaliou 831 crianças de 7 a 12 anos (idade média de 9,3 anos) e que mostrou incidência de cinetose maior no sexo femino, avaliando 831 crianças de 7 a 12 anos (idade média de 9,3 anos) ${ }^{9}$, corrobora com os dados do presente estudo. Este mesmo estudo demonstrou uma prevalência crescente de cinetose até a idade de 9 anos (pico), seguida de uma diminuição não linear da prevalência, ${ }^{9}$ assim como os resultados apresentados nesta pesquisa. Adicionalmente, a cinetose tende a aumentar com a idade, e aos 10 anos, as crianças não diferem dos adultos, segundo Chang et al. ${ }^{22}$ Isso pode ser explicado pelo fato de que a coordenação das respostas posturais e a eficiência funcional do sistema vestibular se desenvolvem entre 10 e 15 
anos de idade. ${ }^{23,24}$

No presente estudo, os veículos onde as crianças apresentaram maiores queixas de cinetose foram ônibus e vans, seguidos de carros, sendo que nos outros meios de transportes não foram relatadas presença de cinetose (trêns, aviões, barcos pequenos, balsas ou navios) possívelmente pelo fato de poucas crianças já terem experimentado esses tipos de transportes, já que não são de uso frequênte na região estudada. O gira-gira de parquinhos foi o mais incidente no quesito entretenimento, seguido de brinquedos de parques de diversão. Nossos resultados são consistentes com outros achados relatados na literatura. Um estudo que avaliou 38 escolares, sendo 18 meninas e 20 meninos, com idade entre nove e dez anos, os meios de transporte com prevalência mais alta de cinetose foram o ônibus/van com 42,10\% e o carro com $39,47 \%$, o gira-gira foi o entretenimento com maior prevalência de cinetose com $40,54 \% .^{18}$ Em outro estudo a susceptibilidade à cinetose também foi maior em carros $(43,4 \%)$ e em ônibus $(43,2 \%)$, sendo que essas duas taxas de prevalência foram praticamente equivalentes. ${ }^{9}$ Um estudo de revisão demostrou que, a ocorrência de doença de movimento em carros e transporte rodoviário público parece ser principalmente influenciada pela qualidade do condutor e pela condição da estrada, ambos fatores que determinam oscilações laterais. ${ }^{25}$

As alterações de equilíbrio encontradas nas crianças avaliadas podem estar relacionadas ao processo de maturação do sistema vestibular. Segundo alguns autores, o sistema vestibular desempenha um papel importante no fornecimento de informação para o sistema de equilíbrio. ${ }^{26} \mathrm{O}$ controle postural é uma capacidade complexa composta pela orientação postural e a manutenção do equilíbrio, que resulta de informações geradas a nível central, relacionadas a mecanismos aferentes visuais, vestibulares e somatossensitivos. ${ }^{27}$ O sistema vestibular é responsável pela integração dessas informações, determinando a resposta motora ideal às informações recebidas e originadas das demandas do ambiente, ${ }^{28}$ garantindo equilíbrio e movimento harmônico do corpo por meio da contração e/ou relaxamento dos grupos musculares agônicos/antagônicos (reflexos posturais). ${ }^{29}$

Um estudo que avaliou 147 crianças demonstrou que, entre 6 e 12 anos, as crianças apresentam maturação de ambas as vias vestibulo-oculares e da estratégia de organização sensorial no controle de equilíbrio. Estes resultados implicam que o sistema vestibular e o sistema oculomotor são altamente funcionais, mas ainda estão em maturação ao longo desta faixa etária. ${ }^{30}$

A experiência de cinetose muitas vezes é acompanhada (ou seguida) por instabilidade visível no controle do corpo: os sofredores podem estar visivelmente instáveis em sua postura e marcha. Diferenças no controle postural existem antes do início de qualquer dos sintomas subjetivos e, de fato, antes que os participantes estejam expostos a movimentos experimentais de qualquer tipo. ${ }^{31}$

Não há na literatura atual estudos que avaliaram se existe uma correlação entre a cinetose e o desenvolvimento motor em todos os seus aspectos. Contudo, um estudo demonstrou uma correlação significativa entre o escore do MSSQ-short e todos os testes de equilíbrio postural. ${ }^{9}$ No presente estudo, apesar de não haver 
associação estatisticamente significativa entre a presença de cinetose e aspectos do desenvolvimento motor, alguns dos escolares que apresentavam cinetose demonstraram alterações de equilíbrio estático. Desta forma, é possível hipotetizar que crianças com cinetose podem ser mais vulneráveis a uma possível queda. 9,22

A motricidade global relaciona-se à movimentação dinâmica corporal, envolvendo um grupo de movimentos coordenados de amplos grupos musculares. ${ }^{20}$ A motricidade global está diretamente relacionada com o equilíbrio, o que pode explicar o fato de alguns dos escolares, que apresentavam cinetose, manifestarem alterações neste aspecto. Os demais testes de desenvolvimento motor foram realizados corretamente pela maioria das crianças avaliadas.

As limitações do estudo incluem o fato de que a pesquisa ocorreu em apenas uma escola e desta, poucos pais e/ou responsáveis autorizaram a participação dos alunos, dificultando as análises de associação, assim como a possibilidade de extrapolação dos dados.

\section{CONCLUSÃO}

Foi possível verificar que a maioria das crianças do estudo apresentaram cinetose, destas, a idade mais frequente foi a de 8 anos e o sexo predominante foi 0 feminino. Os meios de transporte que mais obtiveram queixas de cinetose foram ônibus e vans, seguidos de carros. O gira-gira de parquinhos foi o mais incidente no quesito entretenimento, seguido de brinquedos de parques de diversão.

Não foi possível estabelecer se há uma associação entre a presença de cinetose e alterações do desenvolvimento motor, embora tenham sido demonstradas alterações nos aspectos de equilíbrio e motricidade global, uma vez que não foi possível obter uma significância estatística. Desta forma, estudos com ampliação da amostra se fazem necessário para comprovação dos resultados estabelecidos.

\section{REFERÊNCIAS}

1. Catanzariti JF, Guyot MA, Massot C, Khenioui H, Agnani O, Donzé C. Evaluation of motion sickness susceptibility by motion sickness susceptibility questionnaire in adolescents with idiopathic scoliosis: a case-control study. Eur Spine J. 2016;25(2):438-43.

2. Brandt $T$, Daroff RB. The multisensory physiological and pathological vertigo syndromes. Ann Neurol. 1980;7(3):195-203.

3. Schmäl F. Neuronal Mechanisms and the Treatment of Motion Sickness. Pharmacology. 2013;91(3-4):229-41.

4. Wasiak J, Spinks A, Bernath V, Villanueva E. Scopolamine (hyoscine) for preventing and treating motion sickness. Cochrane Database Syst Rev. 2007;18(3):2-26. 
5. Zhang LL, Wang JQ, Qi RR, Pan LL, Li M, Cai YL. Motion sickness: current knowledge and recent advance. CNS Neurosci Ther. 2016;22(1):15-24.

6. Lawther A, Griffin MJ. Prediction of the incidence of motion sickness from the magnitude, frequency, and duration of vertical oscillation. J Acoust Soc Am. 1987;82(3):957-66.

7. Golding JF. Motion sickness susceptibility. Auton Neurosci. 2006;129(1-2):67-76.

8. Klosterhalfen S, Kellermann S, Pan F, Stockhorst U, Hall G, Enck P. Effects of ethnicity and gender on motion sickness susceptibility. Aviat Space Environ Med. 2005;76(11):1051-7.

9. Henriques IF, Oliveira DW, Oliveira-Ferreira F, Andrade PM. Motion sickness prevalence in school children. Eur J Pediatr. 2014;173(11):1473-82.

10.Turner M, Griffin MJ. Motion sickness in public road transport: passenger behavior and susceptibility. Ergonomics. 1999;42(3):444-61.

11.Buyukl F, Tarhan E, Ozluoglu L. Vestibular functions in motion sickness susceptible individuals. Eur Arch Otorhinolaryngol. 2009;266(9):1365-71.

12.Benson AJ. Motion sickness. In: Nicholson AN, Rainford DS, Ernsting J (eds) Aviation Medicine. 3ㄹa ed. Oxford: Butterworth-Heinemann; 1999.

13.Femia P, Pino BG, Perez-Fernandez N. Vestibular examination of children with alterations in balance (I): clinical and instrumental examination methods. Acta Otorrinolaringol Esp. 2011; 62(4):311-317.

14.Miranda LP, Resegue R, Figueiras ACM. A criança e o adolescente com problemas do desenvolvimento no ambulatório de pediatria. J Pediatr. 2003;79(1):33-42.

15. Strauss RS, Pollack HA. Social marginalization of overweight children. $\underline{\text { Arch Pediatr }}$ Adolesc Med. 2003;157(8):746-52.

16.Reason JT, Brand JJ. Motion sickness. London: Academic Press; 1975.

17.França SR, Branco-Barreiro FCA. Susceptibilidade à cinetose no idoso com doença vestibular. Reces. 2013;5(1):30-5.

18.França SR, Pereza MLVD, Scharlachb RC, Branco-Barreiro FCA. Susceptibilidade à Cinetose em Escolares. Rev. Equilíbrio Corporal Saúde. 2015; 7(2): 47-50.

19.Flanagan MB, May JG, Dobie TG. The role of vection, eye movements and postural instability in the etiology of motion sickness. J Vestib Res. 2004;14(4):335-46.

20.Neto, FR. Manual de avaliação motora.1ํㅡ ed. Porto Alegre: Artmed; 2001. 
Artigo Original

Atenção à saúde

21.Neto FR, Santos APM, Xavier RFC, Amaro KN. A Importância da avaliação motora em escolares: análise da confiabilidade da Escala de Desenvolvimento Motor. Rev Bras Cineantropom Desempenho Hum. 2010; 12(6):422-427.

22.Chang $\mathrm{CH}$, Pan WW, Tseng Y, Stoffregen TA. Postural activity and motion sickness during video game play in children and adults. Exp Brain Res. 2012; 217(2):299_ 309.

23. Hatzitaki V, Zisi V, Kollias I, Kioumourtzoglou E. Perceptual-motor contributions to static and dynamic balance control in children. J Mot Behav. 2002;34(2):161-70.

24. Hsu YS, Kuan CC, Young YH. Assessing the development of balance function in children using stabilometry. Int J Pediatr Otorhinolaryngol. 2009;73(5):737-40.

25.Bertolini G, Straumann D. Moving in a Moving World: A Review on Vestibular Motion Sickness. Front Neurol. 2016;7(14):1-11.

26. Mao HY, Kuo LC, Yang AL, Su CT. Balance in children with attention deficit hyperactivity disorder-combined type. Res Dev Disabil. 2014;35(6):1252-8.

27.Günendi Z, Sepici Dinçel A, Erdoğan Z, Aknar O, Yanpal S, Göğüş F, Atalay F. Does exercise affect the antioxidant system in patients with ankylosing spondylitis. Clin Rheumatol. 2010;29(10):1143-7.

28.Duffau H. Brain plasticity: from pathophysiological mechanisms to therapeutic applications. J Clin Neurosci. 2006;13(9):885-97.

29.Zambenedetti M, Sleifer P, Fiorini AC. Perfil otoneurológico e sintomatológico em pacientes vertiginosos. Distúrb Comum. 2011;23(1): 79-85.

30. Charpiot A, Tringali S, lonescu E, Vital-Durand F, Ferber-Viart C. Vestibulo-ocular reflex and balance maturation in healthy children aged from six to twelve years. Audiol Neurootol. 2010;15(4):203-10.

31. Koslucher F, Haaland E, Stoffregen TA. Sex differences in visual performance and postural sway precede sex differences in visually induced motion sickness. Exp Brain Res. 2016;234(1):313-22. 